\title{
Dual-Focuses Metalens for Copolarized and Cross-Polarized Transmission Waves
}

\author{
Ru Ji, Kejian Chen (D), Yujie Ni, Yanan Hua, Kaiwen Long, and Songlin Zhuang \\ Shanghai Key Laboratory of Modern Optical Systems, Engineering Research Centre of Optical Instrument and System, \\ Ministry of Education, University of Shanghai for Science and Technology, Shanghai 200093, China \\ Correspondence should be addressed to Kejian Chen; ee.kjchen@gmail.com
}

Received 23 January 2018; Accepted 19 March 2018; Published 2 May 2018

Academic Editor: Zhenzhou Cheng

Copyright (C) $2018 \mathrm{Ru}$ Ji et al. This is an open access article distributed under the Creative Commons Attribution License, which permits unrestricted use, distribution, and reproduction in any medium, provided the original work is properly cited.

\begin{abstract}
Metasurfaces can reshape the wavefront in the desired manner by manipulating the phase profile and amplitude of the incident wave. In this paper, we demonstrate an ultrathin terahertz metalens based on our designed resonator structure, where the polarization state can be converted to the orthogonal direction and the parabolic phase profile is designed covering a $2 \pi$ phase region. Many functional metalenses are also engineered to meet the demand of focusing, dual-polarization confocal, and dual focuses for orthogonal polarization in the frequency range from 0.65 to $0.8 \mathrm{THz}$. The presented metalenses can provide potential applications in terahertz communications and imaging systems.
\end{abstract}

\section{Introduction}

Metasurfaces, a category of 2D planar metamaterials, have shown extraordinary capability in arbitrarily controlling electromagnetic waves, enabling novel electromagnetic phenomena such as anomalous refraction/reflection [1,2], polarization conversion [3,4], wave plates [5], flat lenses [6-10], vortex beam [11], and hologram [12]. In comparison with conventional optical components, metasurfaces not only introduce abrupt phase discontinuities in the reflected/transmitted beams but also greatly compress the device thickness. For example, lenses based on metasurfaces (metalenses) can be easily integrated in an optical system to minimize system size and overcome the limitations of conventional lenses. So far, metalenses have been widely studied at the frequency range of optics [6], terahertz [7, 8], and microwave [10]. In a previous study, it has been demonstrated that many kinds of metalenses with single focus can be realized successfully. In 2013, Capasso's group proposed an optical planar lens and axicons using V-shaped resonators [6]. Then, later, the double-focus and multifocus lenses have been paid more and more attention. In 2015, Wang et al. reported that a terahertz metalens employed C-shaped resonators for multiple focusing in the broadband region [8]. In 2016, a terahertz ultrathin lens with multifocusing spots has been proposed using the Yang-Gu algorithm [13]. The abovementioned metalenses have the same characteristics where the cross-polarized waves are focused. Requirements such as dual-polarization focusing, or dual focuses for orthogonal polarization, which have more potential applications [13-16] for terahertz dualpixel imaging and dual-channel communication, could not be fulfilled by the existing metalenses.

In this paper, we proposed several metalenses based on our designed cross-polarization resonator to meet those requirements mentioned above, by arranging the position and orientation of resonators on the metasurface. Finally, $2 \mathrm{D}$ resonator arrays are arranged in a specific order, and the transmission wave under orthogonal polarization states realizes lateral separation. Such proposed metalenses pave a novel way for planar terahertz device designs.

\section{Design of the Metalenses and Simulation}

To independently control the phase and amplitude of the transmitted terahertz wave, we propose a novel resonator structure, a wire connected reticle-like resonator (WCRR) shown in Figure 1(a). The unit cell is made of metal materials, $200 \mathrm{~nm}$ gold $(\mathrm{Au})$ on a polyimide $(\mathrm{PI})$ substrate. Au material was modeled as lossy metal with conductivity of $4.56 \times$ $10^{7} \mathrm{~S} / \mathrm{m}$ [17]. The polyimide film was modeled as lossless dielectric with dielectric constant of 3.5 [18] (thickness ST = $30 \mu \mathrm{m}$ ). The geometric dimensions of WCRR are marked out 

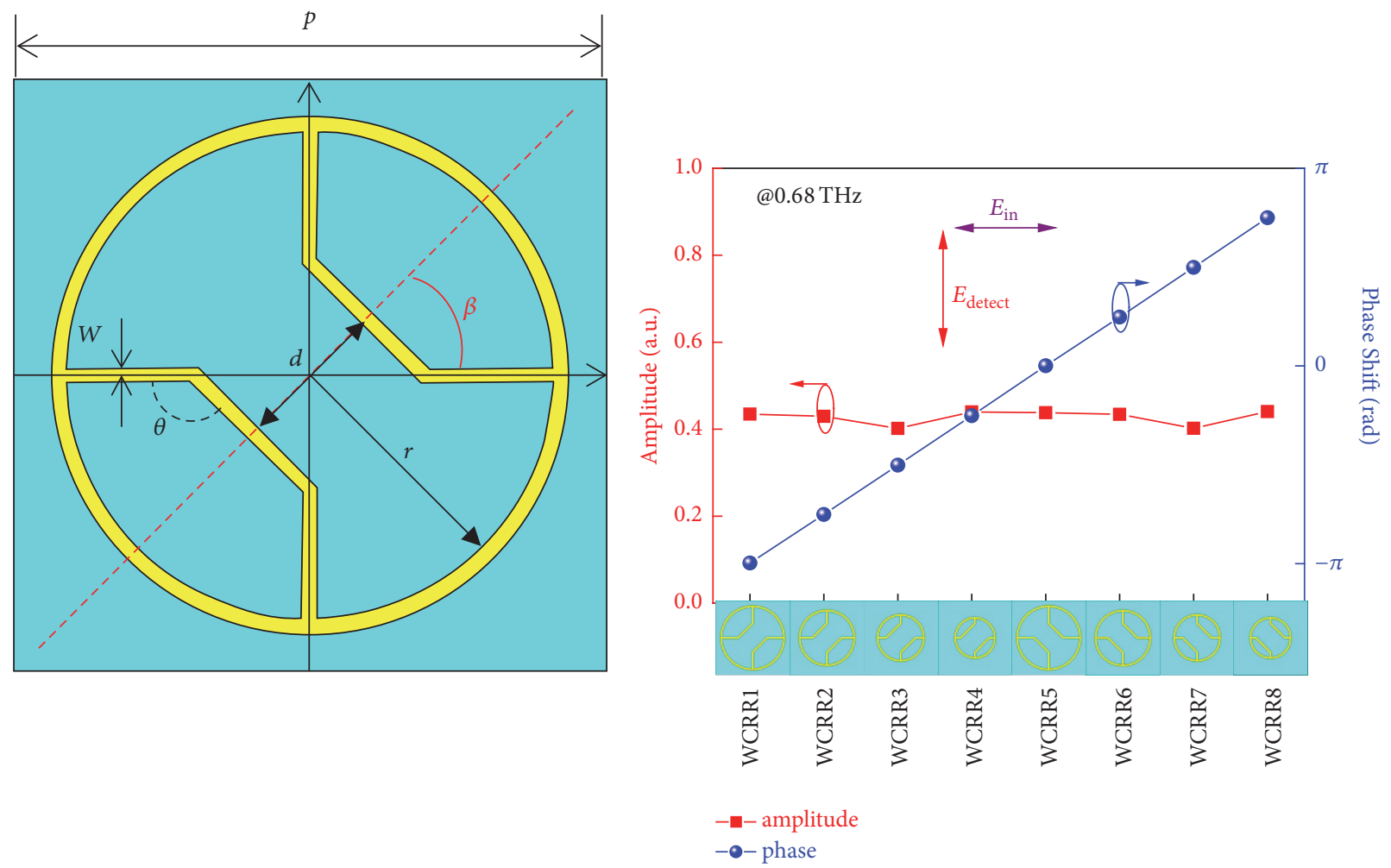

(a)

(b)

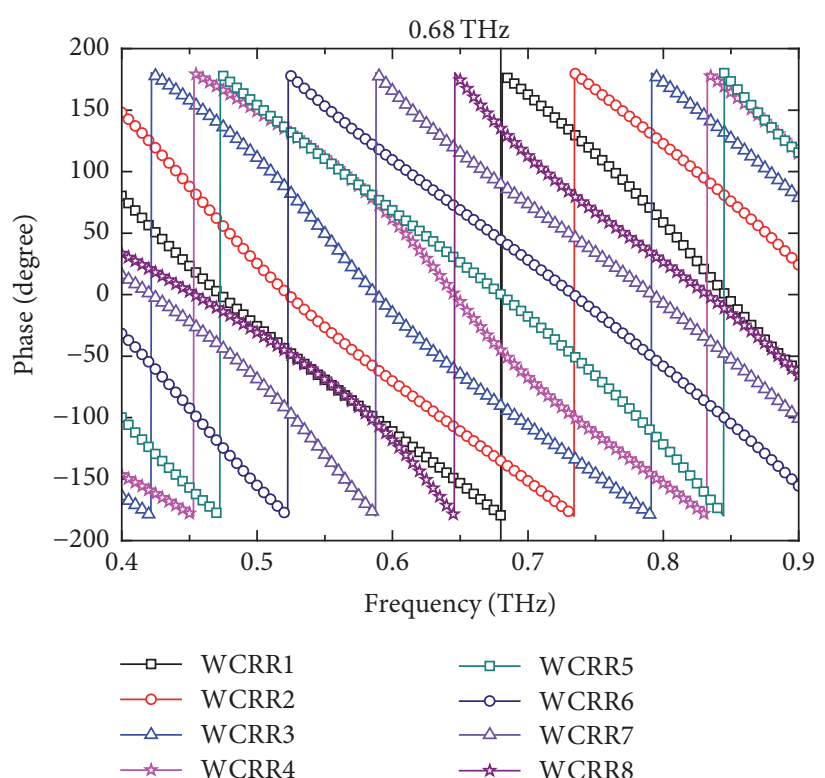

(c)

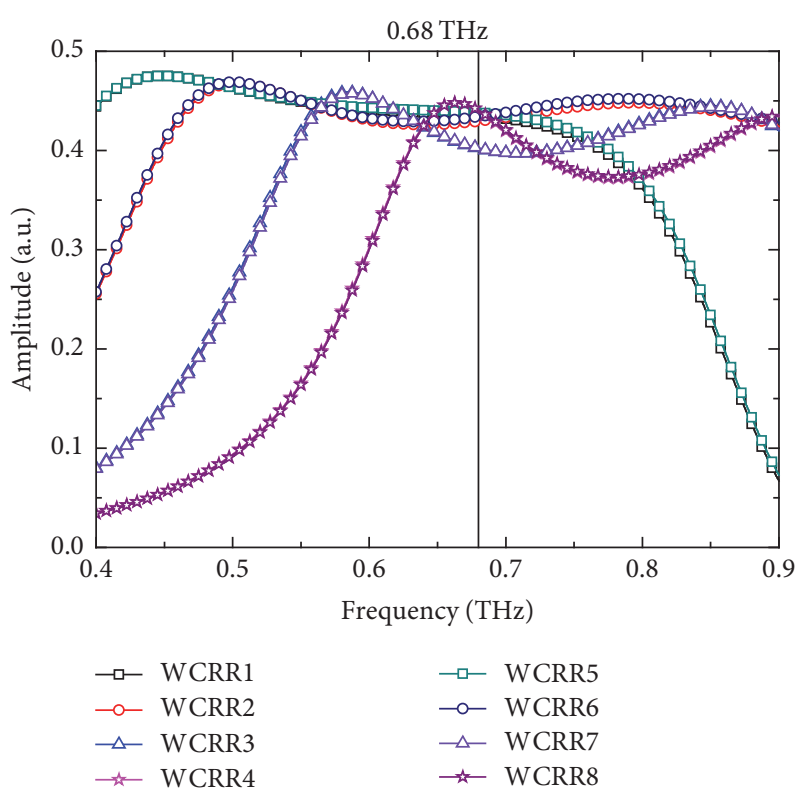

(d)

FIGURE 1: (a) Schematic views of the WCRR. (b) Schematic representation of eight resonators corresponding to eight different phases at $0.68 \mathrm{THz}$. (c, d) Simulated phase shift and transmission amplitude for eight WCRRs, respectively.

as follows: a period $p=200 \mu \mathrm{m}$, wire width $w=5 \mu \mathrm{m}, d=$ $60 \mu \mathrm{m}$, and $\theta=135^{\circ}$. In particular, $r$ denotes the radius of the ring which introduces different abrupt phase shifts when the terahertz wave propagates through the metasurface. To configure the metalens, the commercial software CST Microwave Studio is employed to find out the elementary resonators with specific phase interval distribution and almost identical transmitted amplitude for the cross-polarized waves. For example, as shown in Figure 1(b), four different elementary resonators (WCRR1 WCRR4) were designed with the same width $w=5 \mu \mathrm{m}$ but different radii $r(87.4 \mu \mathrm{m}, 76 \mu \mathrm{m}, 64 \mu \mathrm{m}$, and $56.2 \mu \mathrm{m})$. When the parameter of rotated orientation $\beta$ 


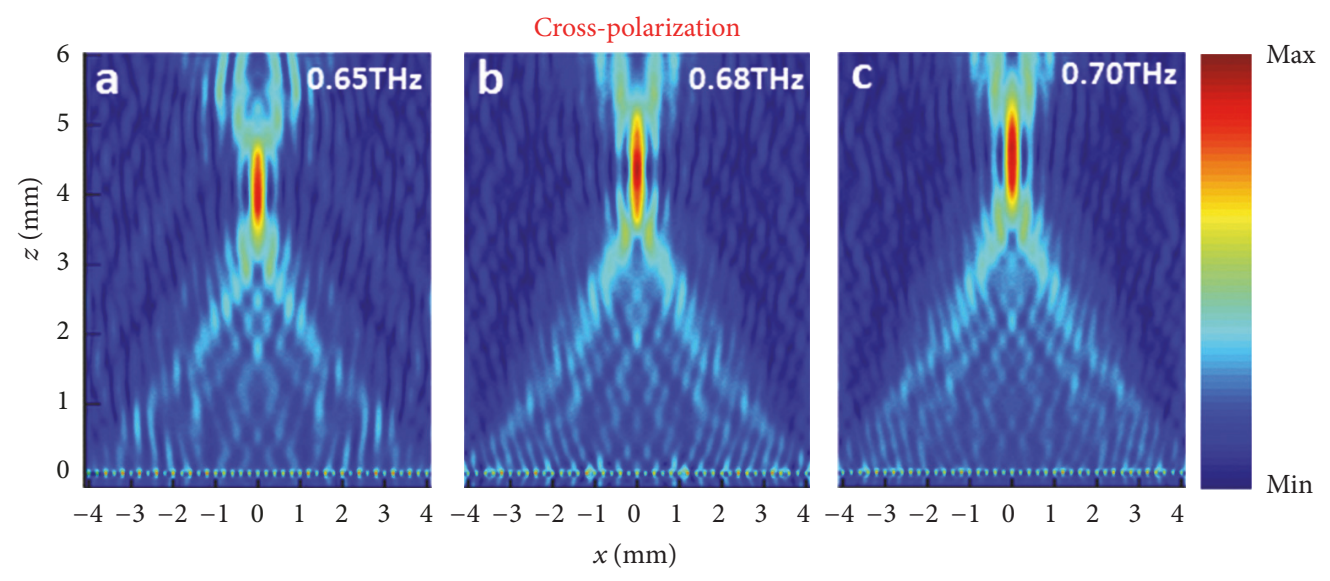

(d)

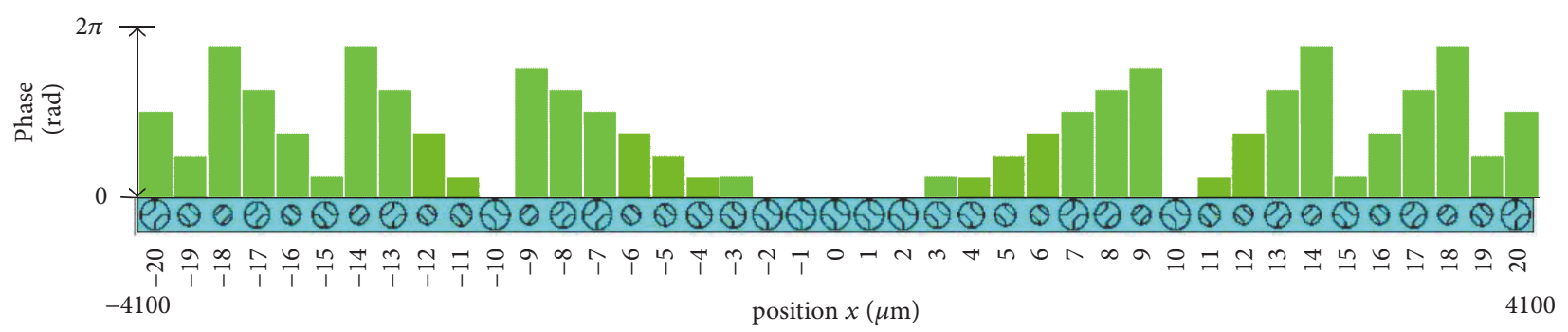

FIgURE 2: $(\mathrm{a}-\mathrm{c})$ The electric field distribution of the cross-polarized transmitted wave for VPFMs at different frequencies. (d) The phase distribution of VPFMs composed of 41 WCRRs.

is set as $-45^{\circ}$, these four resonators, respectively, represent $-\pi,-3 \pi / 4,-\pi / 2$, and $-\pi / 4$ phase at $0.68 \mathrm{THz}$. Following that, the resonators (WCRR $5 \sim$ WCRR 8 ) with $0, \pi / 4, \pi / 2$, and $3 \pi / 4$ phase can be achieved only by flipping WCRR1-WCRR 4 along the $x$-axis. The transmission amplitudes of these eight resonators at $0.68 \mathrm{THz}$ fluctuate around 0.41 slightly, as shown in Figure 1(b). The cross-polarized transmitted phase spectrum and amplitude spectrum for WCRR1 WCRR8 are shown in Figures 1(c) and 1(d). It is obvious that the overlapping frequency band from 0.65 to $0.80 \mathrm{THz}$ can be the appropriate operation region of the metalens. Compared with the demonstrated resonators (e.g., V-shaped [6], C-shaped [8], and double C-shaped [9]), these eight available resonators meet the demands of identical amplitude and phase discontinuity with a step of $\pi / 4$ only by altering the radius $r$. By studying the surface current distributions of the different resonators, we found that when the radius $(r)$ of its outer ring rises, the surface current of the resonator flows past a longer path, which finally makes the corresponding frequency of the abrupt phase $(\pi)$ point become lower. As the phase spectrums stay parallel to each other after phase $(\pi)$ point for different resonators as shown in Figure 1(c), therefore, by adjusting the outer ring of the resonator to change phase $(\pi)$ points, arbitrary phase $(0-2 \pi)$ can be obtained at the fixed operational frequency point. Therefore, our WCRRs provide more flexibility for the design of metalenses. The conventional spherical lenses are satisfied with the parabolic phase profile [10], and the relative phase distribution is expressed by

$$
\Delta \phi(d, F)=\frac{2 \pi}{\lambda}\left(\sqrt{d^{2}+F^{2}}-F\right)
$$

where $d=\sqrt{x^{2}+y^{2}}$ is the distance from point $(x, y)$ to the origin (the center of the metalens), $\lambda$ is the operational wavelength, and $F$ is the desired focal length. When $y$ is zero, spatial phase distribution can be evolved into $1 \mathrm{D}$ flat metalens. In this paper, 41 resonators are combined into $1 \mathrm{D}$ metalens with parabolic phase profile as shown by the symbols in Figure 2(d) at the operational frequency of $0.68 \mathrm{THz}(\lambda \approx 441 \mu \mathrm{m})$. These WCRRs are individually identified with the numeric from -20 to 20 in order, which are divided into two groups according to odd and even number bits. To evaluate the performance of the focusing effect, the focal distance of the designed metalens is set at $F=10 \lambda$ $(4.41 \mathrm{~mm})$. Figure $2(\mathrm{~b})$ shows the electric field intensity of the cross-polarized transmitted wave under normal $x$-polarized $\mathrm{THz}$ wave incidence (horizontally polarized state), which clearly proves that the electric field intensity reaches the peak at $4.36 \mathrm{~mm}$ away from the interface of the metalens. This proves that the theoretical calculation results and CST simulation results are in good consistency. And it has to be mentioned that the electric field intensity at the focal spot reaches 2.4 times the incident electric field intensity. From Figures 2(a)-2(c), it can be clearly seen that the focal spot is farther when the frequency is higher. This vertically polarized focusing metalens (VPFM) for cross-polarization transmission wave has different focuses at the operational frequency band, within a range from 3.93 to $5.76 \mathrm{~mm}$. 

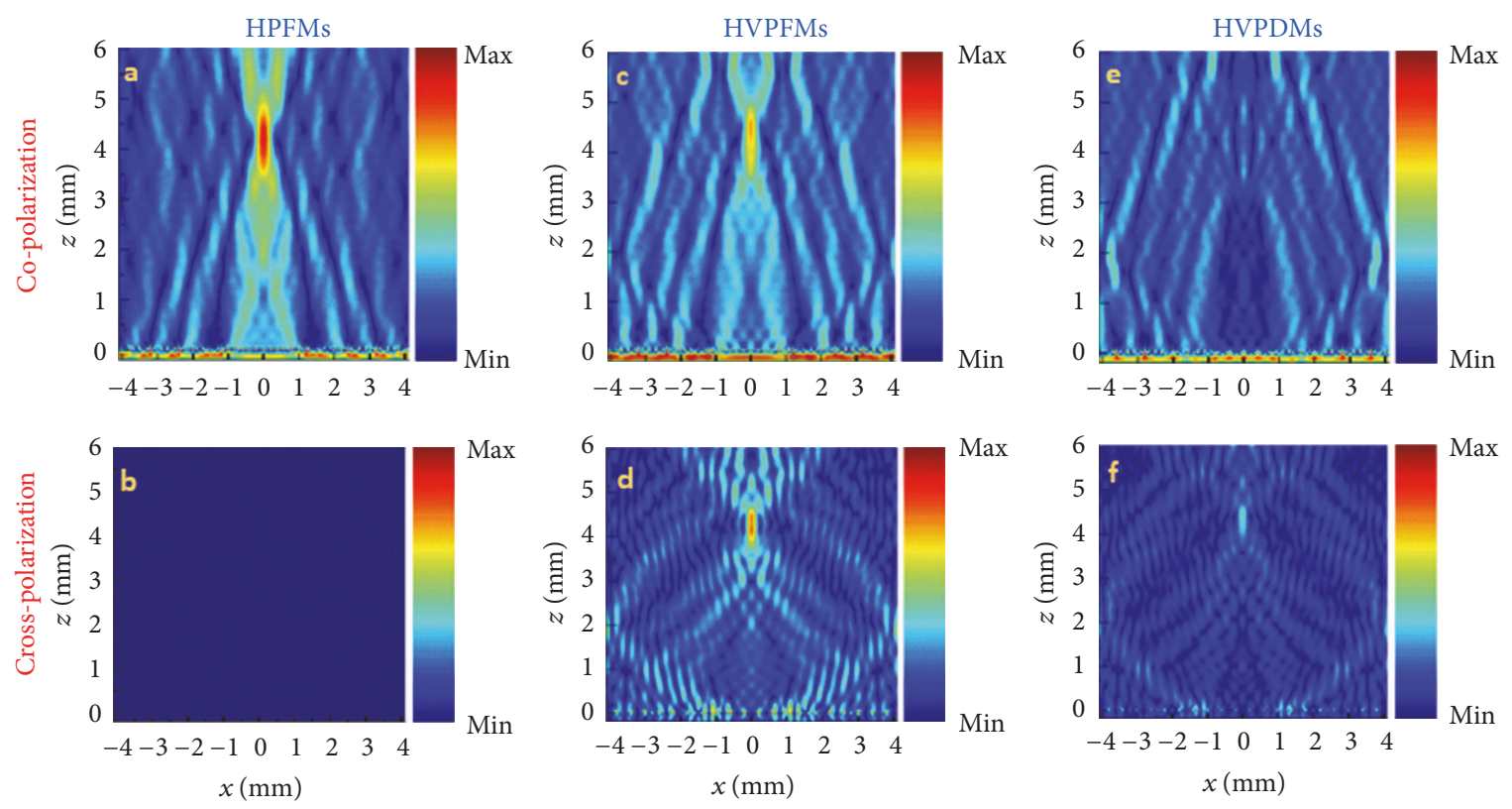

(g)

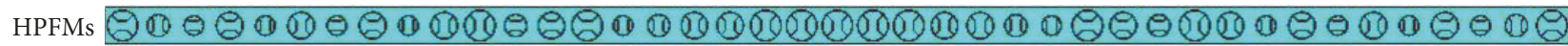

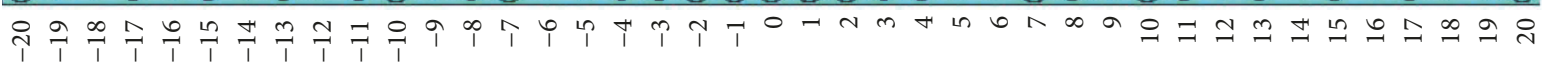

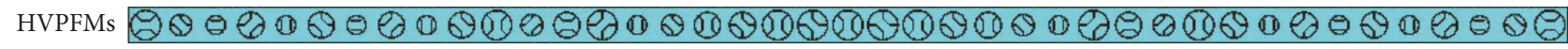

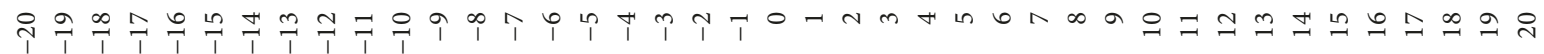

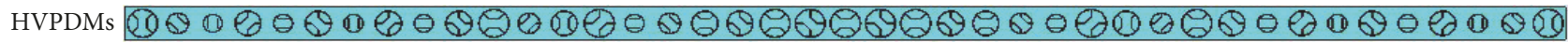

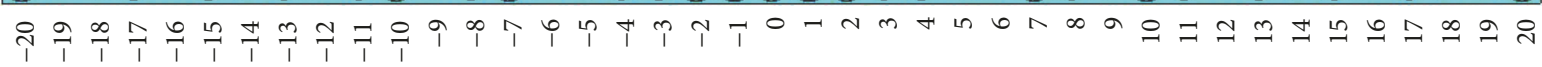

FIGURE 3: (a-f) The electric field intensity distribution of copolarized transmitted wave and cross-polarized transmitted wave at $0.68 \mathrm{THz}$ for HPFMs, HVPFMs, and HVPDMs, respectively. (g) Structures of these three metalenses.

When the WCRRs in VPFMs are rotated clockwise by $45^{\circ}$ around the center of themselves, horizontally polarized focusing metalenses (HPFMs) are formed, as shown in Figure 3(g). Compared to the VPFMs, the cross-polarized polarized transmission amplitude of those WCRRs in HPFMs drops close to zero, so the cross-polarized focusing effect disappears, as shown in Figure 3(b). Although the copolarized transmission amplitude of WCRRs in HPFMs is not uniform, the phase profile is analogous to the phase distribution of the cross-polarized focusing effect [5], which finally realizes focusing effect for copolarization transmission wave. So, the copolarized/cross-polarized distribution of the electric field intensity, as shown in Figures 3(a) and 3(b), is completely different from that in Figure 2(b). However, at the copolarized direction $\left(E_{x}\right)$, HPFMs yield focusing effect (focal length of $F=4.2 \mathrm{~mm}$ ), as shown in Figure 3(a). Therefore, metalenses with WCRRs can transform functionally from crosspolarized focusing to copolarized focusing by independently rotating the unit resonators.

Again, when the entire even-numbered bit resonators of the VPFMs are clockwise rotated in situ by $45^{\circ}$, a special metalens named HVPFM is formed. HVPFMs make both the copolarized and the cross-polarized transmitted wave converge in the identical location for normal incidence of horizontal polarized $\left(E_{x}\right)$ wave at $0.68 \mathrm{THz}$, as shown, respectively, in Figures 3(c) and 3(d). The focusing of the crosspolarized transmitted terahertz wave is mainly attributed to the even bit unit WCRR, which is based on the same principle as VPFMs' mechanism (Figure 2(b)). And the principle of the focusing under the copolarized transmitted wave is consistent with HPFMs' mechanism. HVPFM realizes focusing effect in both the copolarized and the cross-polarized direction at the same point, so the linearly polarized terahertz plane wave can be transformed into circular wave on the focused point. It synthesizes the functionality of a quarter-wave plate and a focusing lens in an ultrathin metalens.

In addition, when the entire even-numbered bit WCRRs of the VPFMs are counterclockwise rotated in situ by $45^{\circ}$, another metalens (named HVPDM, shown in Figure $3(\mathrm{~g})$ ), with a slight extinction effect (Figures 3(e) and $3(\mathrm{f})$ ) can be achieved. Since the phase of WCRRs on the even-numbered bits in HVPDMs is increased by a certain phase shift compared with those in VPFMs, the formed coherent cancellation effect in this device finally realizes the extinction phenomenon. Therefore, the WCRRbased metalens has the potential ability to switch between 


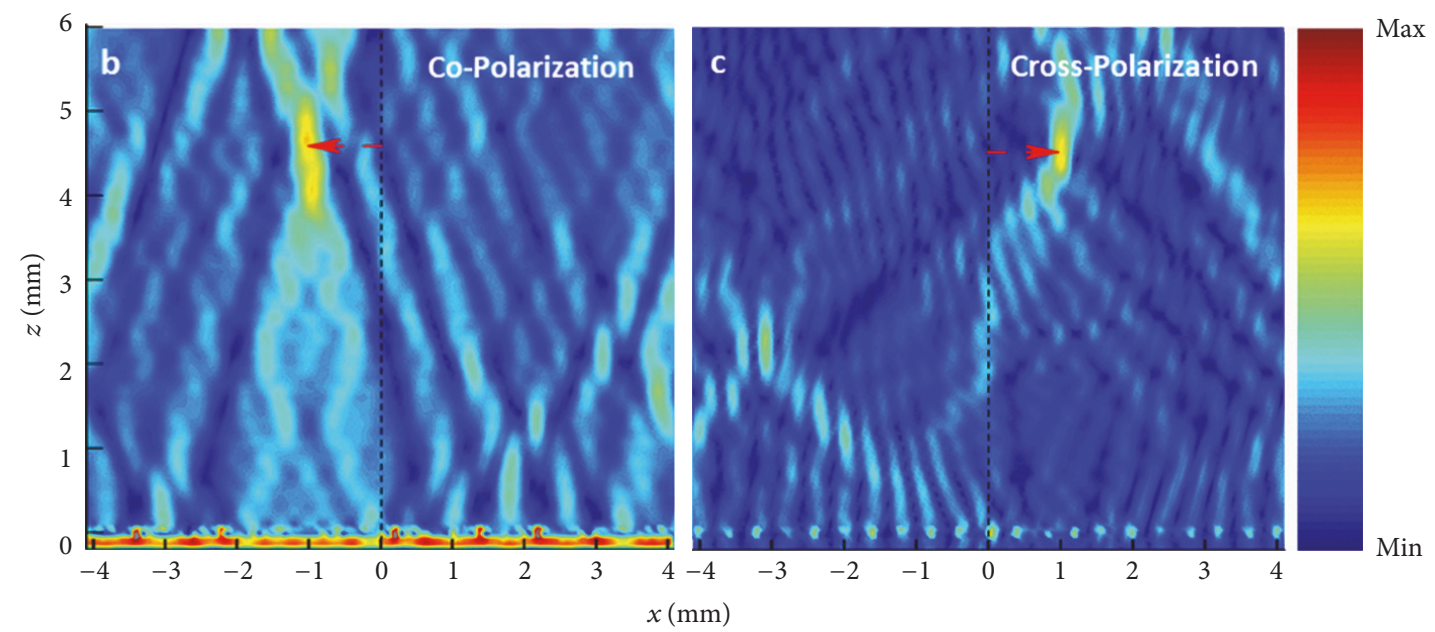

(a)

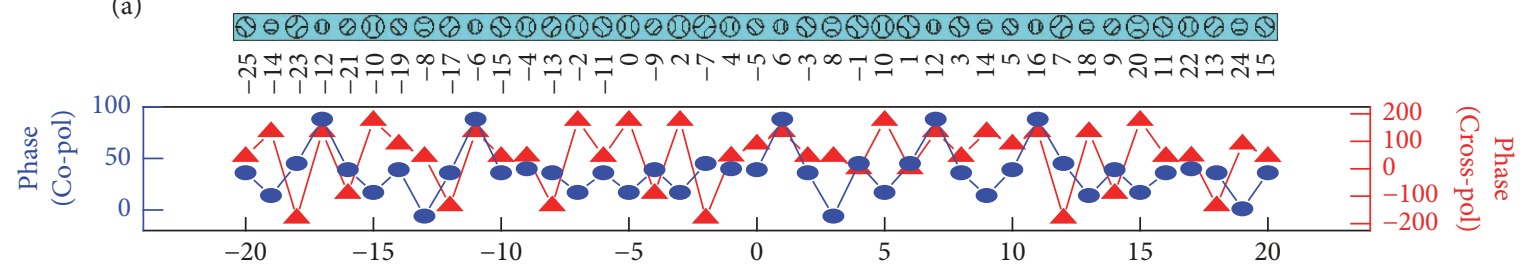

FIgure 4: (a) The structure of SOPFM and its phase distributions at $0.68 \mathrm{THz}$. (b, c) The electric field intensity distribution of copolarized and cross-polarized transmitted wave, respectively.

focusing and extinction freely by rotating even-numbered bit WCRRs.

Finally, the odd-numbered and the even-numbered WCRRs from the HVPFMs are shifted, respectively, to the right and left by five times the periodic length to create a new metalens (named SOPFMs) as shown in Figure 4(a). With this metalens, the transverse distance of the focal spots of two orthogonal polarization states is about 10-bit units $(2 \mathrm{~mm})$, as shown in Figures 4(b) and 4(c). On account of the WCRRs of this metalens that are arranged asymmetrically, the focal spots of two outgoing polarized directions are slightly distorted (Figure 4(a)). But the focal spots still exist, and the linear polarized plane waves can still be focused on the separated focal positions at the copolarized and cross-polarized directions, respectively. Such kind of focusing separation in copolarized and cross-polarized direction can be applied in several fields, such as dual-focuses imaging and communication.

For the practical usage of metalens, a 2D device structure is needed. Here, the $1 \mathrm{D}$ resonator array is expanded to 31 $\times 31$ resonator arrays (with a size of $3.1 \times 3.1 \mathrm{~mm}^{2}$ ), as shown in Figure 5. In 2D situation, the effect of the losses of material becomes more obvious and cannot be ignored for normal materials. Even the PI material is a low loss material, and the loss tangent $\delta=0.0057$ of the polyimide [16] is taken into account in the following $2 \mathrm{D}$ structure simulation. It is important to mention that when the loss tangent is set to 0.0057 , the phase spectrum of the crosspolarized transmittance of the WCRR is almost not shifted compared to the WCRR without loss (Figure 1(c)). And its

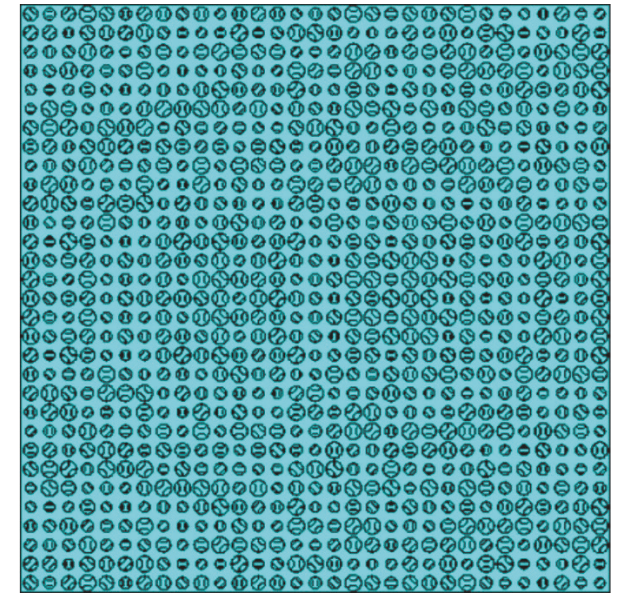

Figure 5: Photograph of the designed 2D device structure.

amplitude only decreases less than one hundredth that of WCRR in Figure 1(d). Figures 6(a) and 6(b) show the electric field intensity of those two focusing spots of orthogonal polarization transmission waves with $2 \mathrm{~mm}$ distance. For the co-/cross-polarized state, the focal length of these two focal spots along the horizontal orientation is identical $(4.23 \mathrm{~mm})$ in the $x-o-z$ planes, the electric field amplitude reaches their maximum, respectively, and electric field intensity deviation ratio (EFIDR $\left.=\left|E_{\text {co }}-E_{\text {cross }}\right| /\left|E_{\text {co }}+E_{\text {cross }}\right|\right)$ is 0.02 , which proves that the focusing property of these two focal spots is uniform. The sizes of these two focal spots along the vertical plane of 

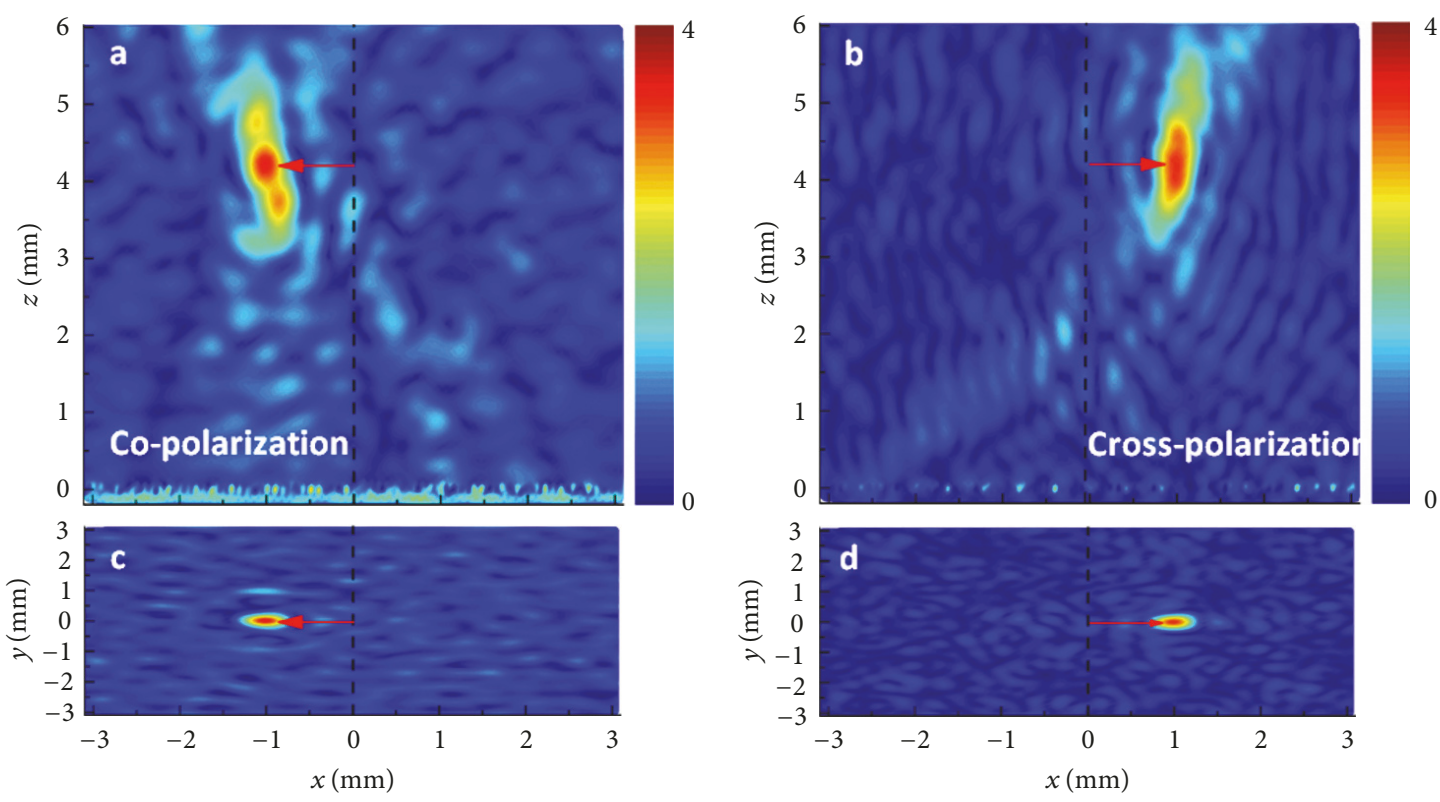

Figure 6: $(\mathrm{a}, \mathrm{b})$ The copolarized and cross-polarized electric field intensity of the transmitted waves in the $x-o-z$ plane at $0.68 \mathrm{THz}$, respectively. (c, d) The copolarized and cross-polarized electric field intensity of the transmitted waves in the $x$-o-y plane at $Z=4.23 \mathrm{~mm}$.

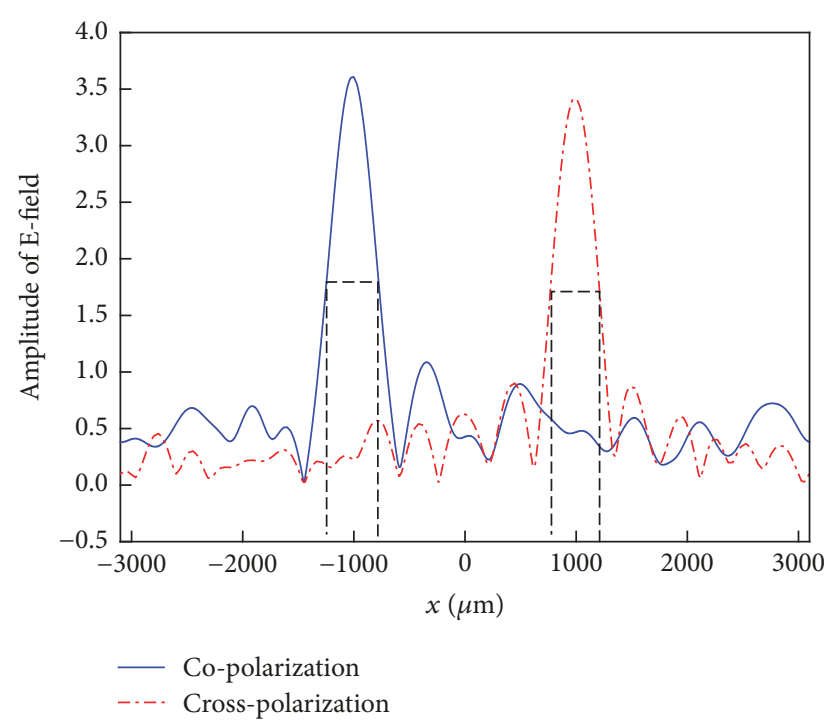

Figure 7: Electric field amplitude in the $X$ direction (extracted at $Z$ corresponding to the focal points) for copolarization and crosspolarization waves, respectively.

the incident wave are also uniform as shown in Figures 6(c) and $6(\mathrm{~d})$.

To evaluate the focusing effect, the amplitude of E-field along the $X$ direction is extracted from Figure 6 at the focal plane $(Z=4.23 \mathrm{~mm})$, as shown in Figure 7. The calculated spot sizes (the full width at half maximum (FWHA) of the focus beam width) of copolarization and cross-polarization waves are $470 \mu \mathrm{m}$ [from $-1245 \mu \mathrm{m}$ to $-775 \mu \mathrm{m}$ ] and $440 \mu \mathrm{m}$ [from $770 \mu \mathrm{m}$ to $1210 \mu \mathrm{m}$ ], respectively. The focusing performance is acceptable. As the dual focuses for orthogonal polarization can be designed independently, as shown in
Figure 6, such kind of dual-focus metalens can be applied in some potential fields, such as dual-focus imaging and duplex communication systems.

\section{Conclusions}

In summary, we have presented a novel resonator structure design (WCRR) in this paper. It can manipulate the phase of the terahertz wave freely only by adjusting the radius size of the resonator. The metalens composed of the WCRRs can converge the transmission waves, achieving the phenomenon of focusing and extinction in two orthogonal polarization directions, and realize the horizontal separation of the focal points of the outgoing wave in two orthogonal polarization directions. These metalenses provide superior technical support for practical applications including imaging and communications.

\section{Conflicts of Interest}

The authors declare that they have no conflicts of interest.

\section{Acknowledgments}

This study was supported by the National Natural Science Foundation of China (no. 61205095) and the Shanghai Young College Teacher Develop funding schemes (no. slg11006.).

\section{References}

[1] N. Yu, P. Genevet, M. A. Kats et al., "Light propagation with phase discontinuities: Generalized laws of reflection and refraction," Science, vol. 334, no. 6054, pp. 333-337, 2011. 
[2] S. L. Sun, Q. He, S. Y. Xiao, Q. Xu, X. Li, and L. Zhou, "Gradientindex meta-surfaces as a bridge linking propagating waves and surface waves," Nature Materials, vol. 11, no. 5, pp. 426-431, 2012.

[3] N. K. Grady, J. E. Heyes, D. R. Chowdhury et al., "Terahertz metamaterials for linear polarization conversion and anomalous refraction," Science, vol. 340, no. 6138, pp. 1304-1307, 2013.

[4] L. Li, Y. Li, Z. Wu, F. Huo, Y. Zhang, and C. Zhao, "Novel polarization-reconfigurable converter based on multilayer frequency-selective surfaces," Proceedings of the IEEE, vol. 103, no. 7, pp. 1057-1070, 2015.

[5] N. Yu, F. Aieta, P. Genevet, M. A. Kats, Z. Gaburro, and F. Capasso, "A broadband, background-free quarter-wave plate based on plasmonic metasurfaces," Nano Letters, vol. 12, no. 12, pp. 6328-6333, 2012.

[6] F. Aieta, P. Genevet, M. A. Kats et al., "Aberration-free ultrathin flat lenses and axicons at telecom wavelengths based on plasmonic metasurfaces," Nano Letters, vol. 12, no. 9, pp. 4932-4936, 2012.

[7] D. Hu, X. Wang, S. Feng et al., "Ultrathin Terahertz Planar Elements," Advanced Optical Materials, vol. 1, no. 2, pp. 186-191, 2013.

[8] Q. Wang, X. Zhang, Y. Xu et al., "A broadband metasurfacebased terahertz flat-lens array," Advanced Optical Materials, vol. 3, no. 6, pp. 779-785, 2015.

[9] A. Forouzmand, S. Tao, S. Jafar-Zanjani, J. Cheng, M. Mahdi Salary, and H. Mosallaei, "Double split-loop resonators as building blocks of metasurfaces for light manipulation: Bending, focusing, and flat-top generation," Journal of the Optical Society of America B: Optical Physics, vol. 33, no. 7, pp. 1411-1420, 2016.

[10] A. K. Azad, A. V. Efimov, S. Ghosh, J. Singleton, A. J. Taylor, and H.-T. Chen, "Ultra-thin metasurface microwave flat lens for broadband applications," Applied Physics Letters, vol. 110, no. 22, Article ID 224101, 2017.

[11] N. Kou, S. Yu, and L. Li, "Generation of high-order Bessel vortex beam carrying orbital angular momentum using multilayer amplitude-phase-modulated surfaces in radiofrequency domain," Applied Physics Express, vol. 10, no. 1, Article ID 016701, 2017.

[12] L. Huang, X. Chen, H. Mühlenbernd et al., "Three-dimensional optical holography using a plasmonic metasurface," Nature Communications, vol. 4, article no. 3808, 2013.

[13] J. He, J. Ye, X. Wang, Q. Kan, and Y. Zhang, "A broadband terahertz ultrathin multi-focus lens," Scientific Reports, vol. 6, Article ID 28800, 2016.

[14] R. Appleby and H. B. Wallace, "Standoff detection of weapons and contraband in the $100 \mathrm{GHz}$ to $1 \mathrm{THz}$ region," IEEE Transactions on Antennas and Propagation, vol. 55, no. 11, pp. 29442956, 2007.

[15] E. Cristofani, F. Friederich, S. Wohnsiedler et al., "Nondestructive testing potential evaluation of a terahertz frequencymodulated continuous-wave imager for composite materials inspection," Optical Engineering, vol. 53, no. 3, Article ID 031211, 2014.

[16] Q. Wang, X. Zhang, E. Plum et al., "Polarization and frequency multiplexed terahertz meta-holography," Advanced Optical Materials, vol. 5, no. 14, Article ID 1700277, 2017.

[17] Y. Bai, K. Chen, H. Liu et al., "Optically controllable terahertz modulator based on electromagnetically-induced-transparency-like effect," Optics Communications, vol. 353, pp. 83-89, 2015.
[18] H. Tao, N. I. Landy, C. M. Bingham, X. Zhang, R. D. Averitt, and W. J. Padilla, "A metamaterial absorber for the terahertz regime: design, fabrication and characterization," Optics Express, vol. 16, no. 10, pp. 7181-7188, 2008. 

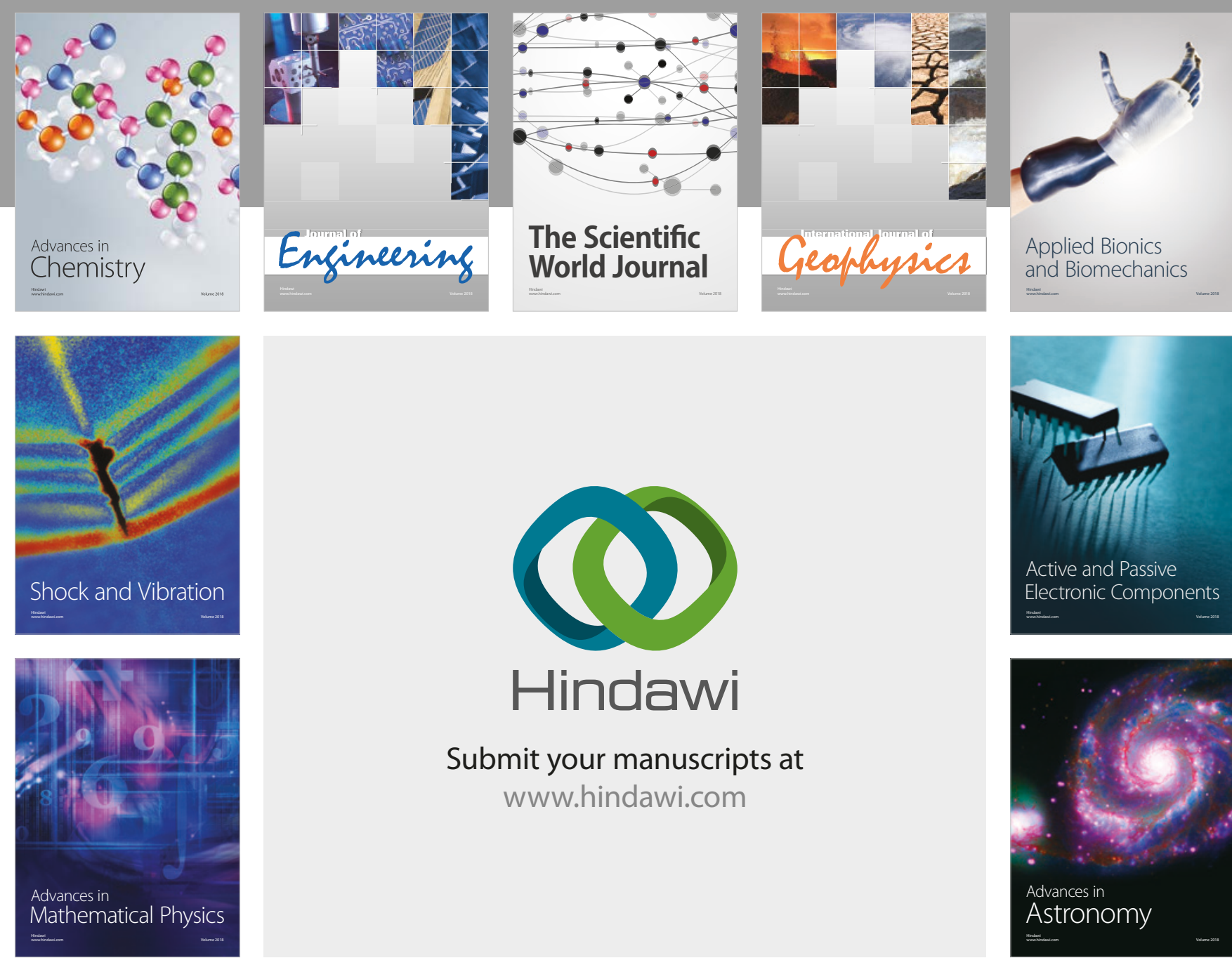

Submit your manuscripts at

www.hindawi.com

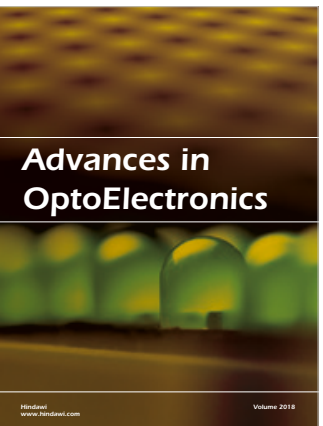

\section{Rotcting Machinery}
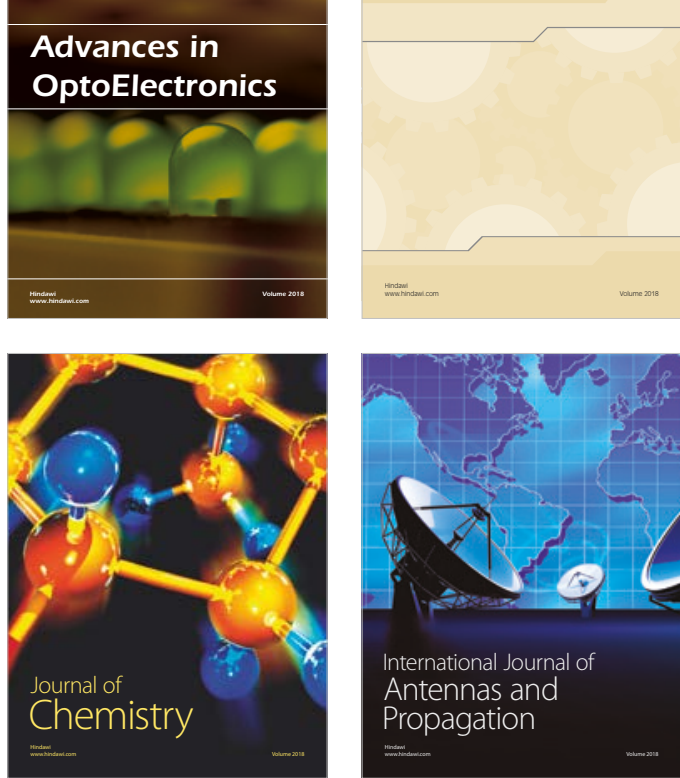

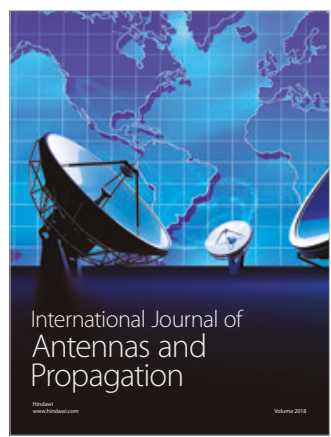

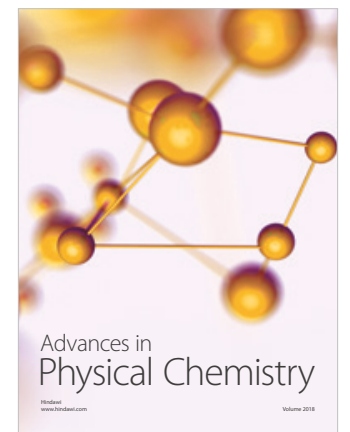

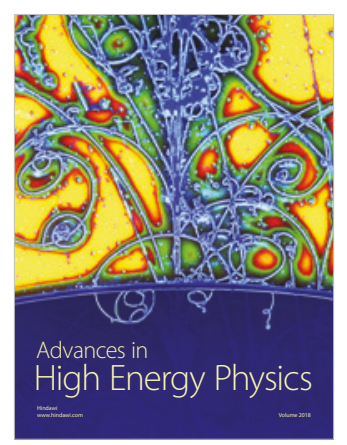

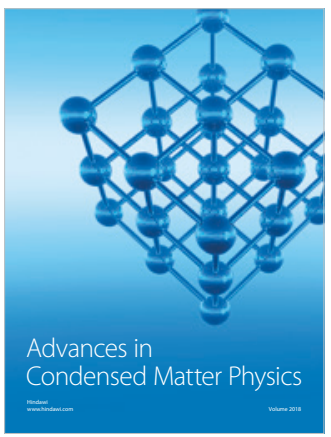

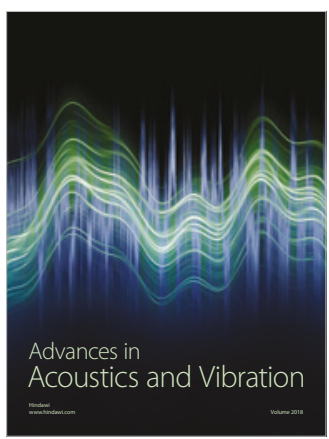

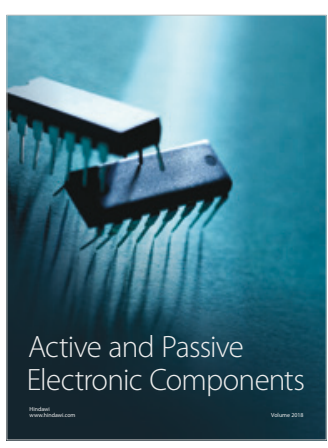
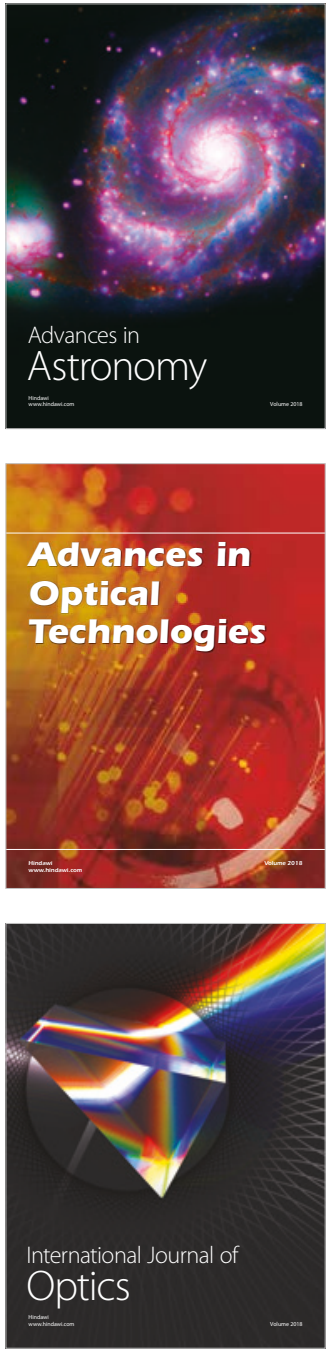